\title{
Placenta Accreta: A Review of the Etiology, Diagnosis, and Management
}

${ }^{1}$ William Goh, ${ }^{2}$ Ivica Zalud

\begin{abstract}
The incidence of placenta accreta is rising, primarily due to the increase in cesarean section rates. The prenatal diagnosis of placenta accretas has been shown to decrease the amount of blood loss and complications. Real-time ultrasound is helpful for diagnosing placenta accreta, and magnetic resonance imaging (MRI) can increase the sensitivity of prenatal diagnosis. Postpartum hysterectomy for placenta accreta has been the standard of therapy for placenta accreta, but conservative management including uterine artery embolization, and leaving the placenta in situ may be considered in patients who want to preserve their fertility. The etiology of placenta accreta is due to a deficiency of maternal decidua, resulting in placenta invasion into the uterine myometrium. The molecular basis is yet to be elucidated, but it probably involves abnormal paracrine or autocrine signaling between the deficient maternal decidua and the invading placenta trophoblastic tissue.
\end{abstract}

Keywords: Accreta, Morbity adherent Placentation, Prenatal diagnosis.

How to cite this article: Goh W, Zalud I. Placenta Accreta: A Review of the Etiology, Diagnosis, and Management. Donald School J Ultrasound Obstet Gynecol 2016;10(3):352-363.

Source of support: Nil

Conflict of interest: None

\section{INTRODUCTION}

There have been tremendous strides made in prenatal care in the last century in the developed world. In the United Kingdom, maternal mortality decreased from 319 per 100,000 live births in 1936 to 15 per 100,000 live births in $1985 .^{1}$ In 1999 , the maternal mortality rate was $13.2 / 100,000$ live births. The decline in maternal mortality has been attributed to a shift to delivering in hospitals, antibiotics, improvements in anesthesia, improvements in blood transfusions and blood products, and access to safe and legal abortion services (Graph 1).

\footnotetext{
${ }^{1}$ Clinical Instructor, ${ }^{2}$ Professor and Chair

1,2Department of Obstetrics, Gynecology and Women's Health John A. Burns School of Medicine, University of Hawaii, Honolulu Hawaii, USA

Corresponding Author: William Goh, Clinical Instructor Department of Obstetrics, Gynecology and Women's Health John A. Burns School of Medicine, University of Hawaii Honolulu Hawaii, USA, Phone: +8089836000, e-mail: wgoh@ hawaii.edu
}

Despite these improvements, hemorrhage continues to be the second most common cause of pregnancyrelated mortality in the United States (Graph 2). ${ }^{2}$

Placenta accretas is a leading cause of postpartum hemorrhage and indication for a gravid hysterectomy. ${ }^{3}$ Traditionally, abnormal placentation has been classified into placenta accreta, placenta increta, and placenta percreta based on the depth of myometrial invasion: Superficial, deep, and through the uterine serosa respectively. The greater the invasion, the greater the risks for hemorrhage and maternal morbidity and mortality. ${ }^{4}$ There has been a substantial increase in the occurrence

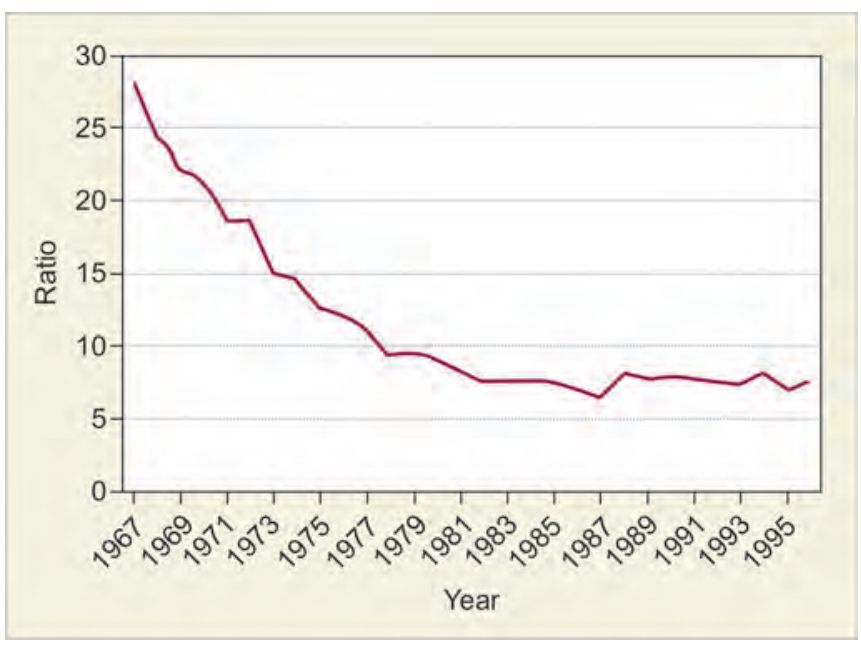

Graph 1: Maternal mortality per 100,000 live births from 1967 to 1995

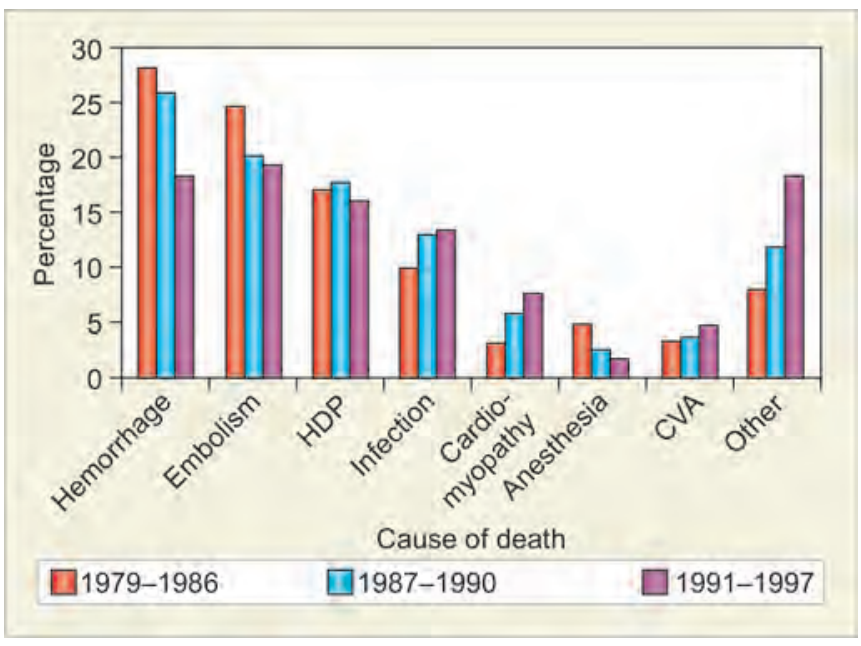

Graph 2: Pregnancy related mortality in the United States, 1991 to 1997 


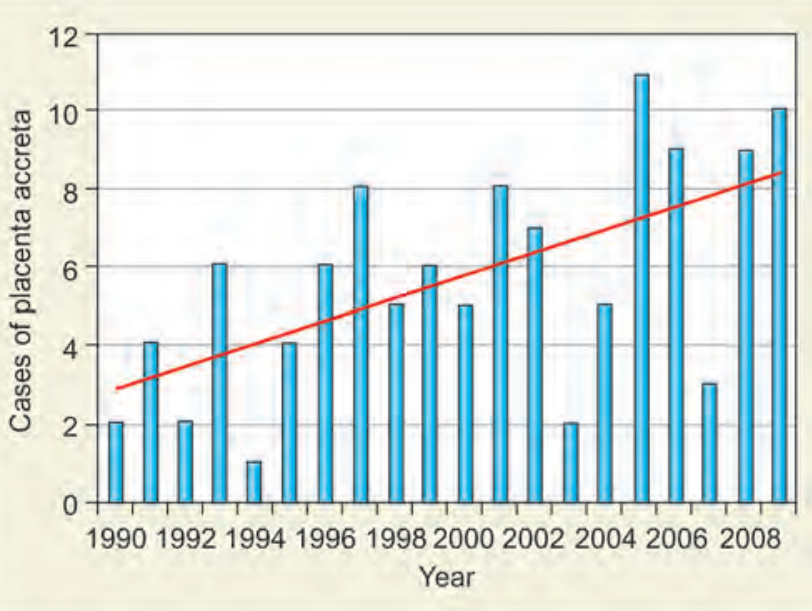

Graph 3: Incidence of placenta accreta at UCSD from 1990 to 2009

of placenta accreta in the last 50 years. It is estimated that there has been a tenfold increase in the rate to around 1:2,500 deliveries. ${ }^{5}$ Warshak et $\mathrm{l}^{6}$ performed a retrospective of all pathologically confirmed placenta accretas at the University of California-San Diego Medical Center from 1990 to 2008 and noted a steady increase in the incidence of abnormal placentation during this time period (Graph 3).

$\mathrm{Wu}$ et $\mathrm{al}^{7}$ looked at 20 years of deliveries $(64,359$ patients) at the University of Chicago. Using histopathological or strong clinical criteria, they calculated an overall incidence of placenta accreta in $1 / 533$ pregnancies. Miller et $\mathrm{al}^{8}$ performed a retrospective analysis of 62 cases of abnormal placentation on cesarean hysterectomy specimens and confirmed that $75 \%$ were placenta accreta, $18 \%$ were placenta increta, and $7 \%$ were placenta percreta (Fig. 1). There are no studies directly comparing outcomes with the depth of uterine invasion, but it is assumed that surgical morbidity is related to the depth of uterine invasion. Zaideh et $\mathrm{al}^{9}$ obtained similar percentages in their retrospective review of abnormal placentation.

\section{Risk Factors for Placenta Accreta}

The most important risk factor for the development of placenta accreta are a prior cesarean delivery and placenta previa in the index pregnancy. ${ }^{3}$ All invasive procedures on the uterus or the uterine cavity have been associated with the subsequent development of placenta accreta. This includes uterine curettage, hysteroscopic surgery, endometrial ablation, uterine artery embolization, and myomectomy. Maternal age is also an independent risk factor for placenta accreta.

The largest prospective study to look at placenta accretas was performed by the maternal-fetal medicine unit (MFMU) network. ${ }^{10}$ This study found that abnormal

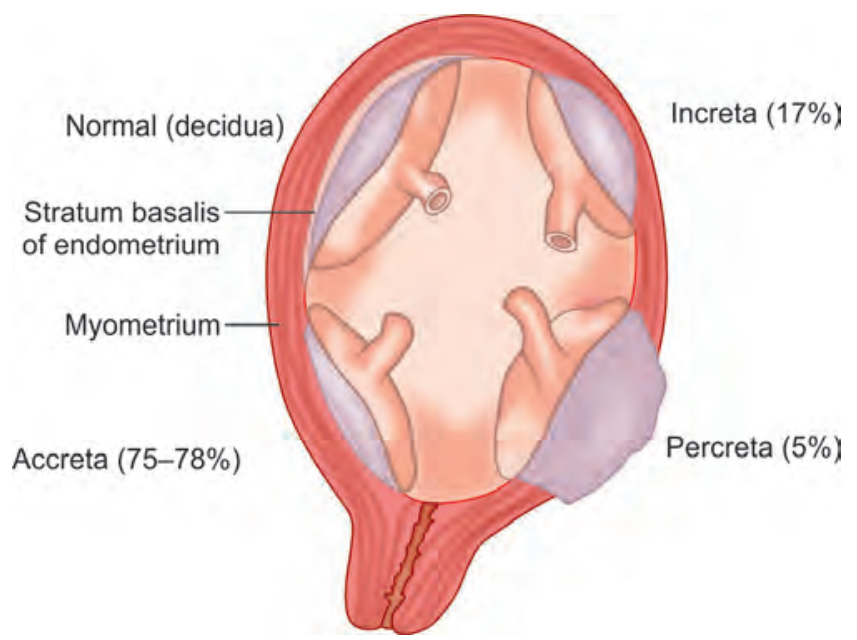

Fig. 1: Placenta accreta implantation sites

Table 1: Risk of abnormal placentation and need for C-hysterectomy by number of previous cesareans

\begin{tabular}{lll}
\hline Cesarean delivery & Abnormal placentation, \% & C-hysterectomy \\
\hline First & 0.2 & - \\
Second & 0.3 & 0.7 \\
Third & 0.6 & 1.4 \\
Fourth & 2.1 & 3.8 \\
Fifth & 2.3 & 5.6 \\
$\geq$ Sixth & 6.7 & 15.2 \\
\hline
\end{tabular}

Table 2: Risk of placenta accreta with placenta previa by number of cesarean deliveries

\begin{tabular}{ll}
\hline Cesarean delivery & Previa-accreta, \% \\
\hline First & 3.3 \\
Second & 11.0 \\
Third & 40.0 \\
Fourth & 61.0 \\
Fifth & 67.0 \\
$\geq$ Sixth & 67.0 \\
\hline
\end{tabular}

implantation increased with each cesarean delivery and increased substantially after the third cesarean (Table 1). The risks of a concomitant cesarean hysterectomy also increased with the diagnosis of placenta accreta (Table 2).

The risk of placenta accreta was markedly increased with previous cesarean deliveries in the presence of a placenta previa

\section{Diagnosis}

\section{Grayscale Ultrasound}

Transabdominal ultrasound is the most common method for the initial diagnosis of placenta accreta. In the first trimester, however, transvaginal ultrasound allows for a more detailed assessment of a placenta's invasiveness. Comstock et $\mathrm{al}^{11}$ performed a retrospective review of 
first-trimester ultrasounds transvaginally and found seven women who were later found to have a placenta accreta (Figs 2 and 3). Six of these women were found to have a low-lying placenta, four of which were abnormally attached to a prior uterine cesarean scar.

Comstock et $\mathrm{al}^{12}$ performed the largest prospective study of grayscale ultrasound for the diagnosis of abnormal placentation. Over 163,000 patients were scanned over a 12-year period, and 2002 had the dual risk factors of a placenta previa or low-lying placenta with a history of a prior cesarean delivery. Thirty-three of these patients had ultrasound findings suspicious for a placenta accreta. These included interruption of the echogenic line at the uterine bladder interface (Fig. 4), placental lacunae (Fig. 5) and obliteration of echolucent area between the uterus and placenta (Fig. 6).

Using these criteria, an ultrasound was labeled positive if any of these criteria were found on at least one ultrasound (Table 3). The sensitivity of ultrasound was $100 \%$, with the presence of placental lacunae having

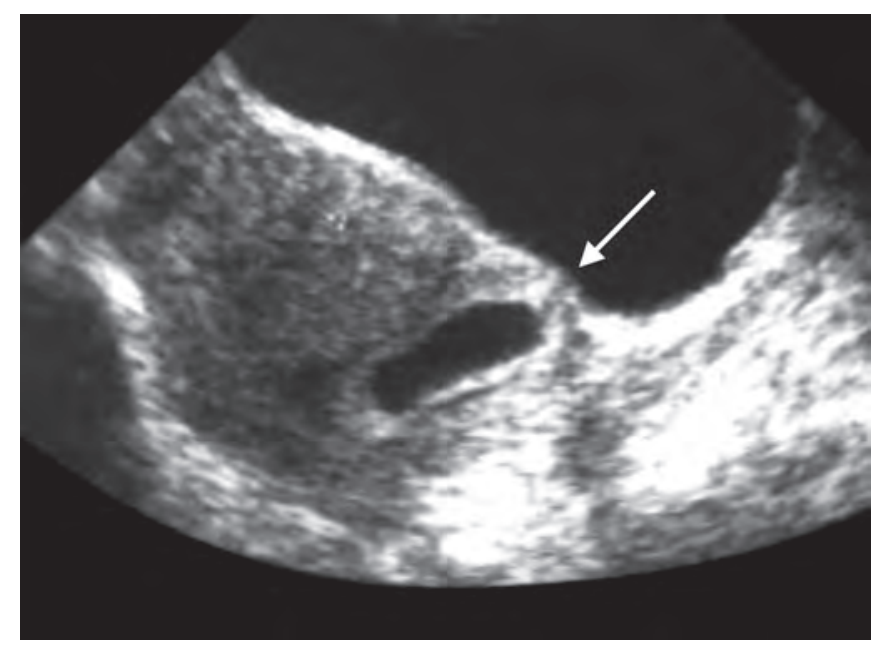

Fig. 3: Placenta percreta diagnosed in the first trimester

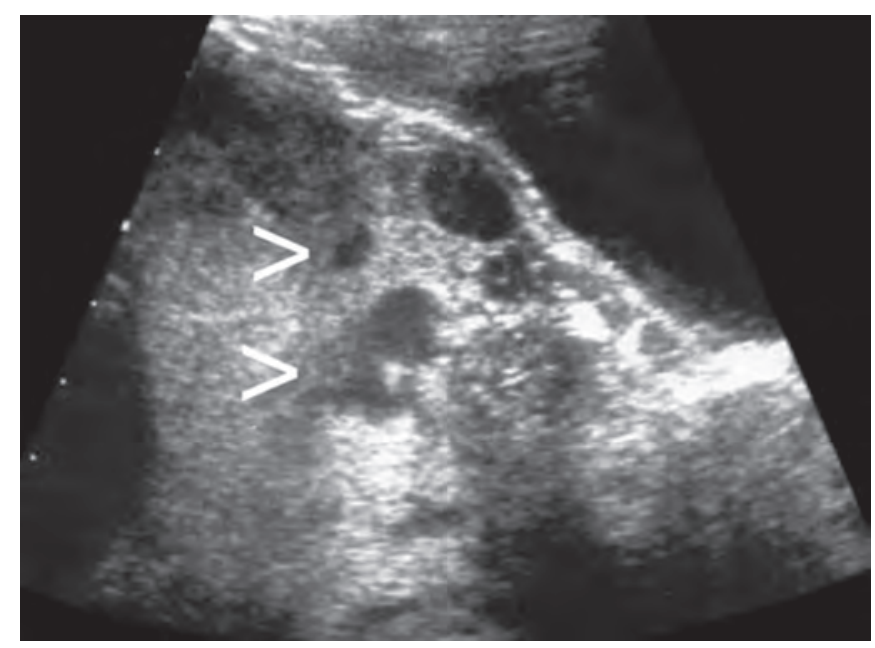

Fig. 5: Placental lacunae the highest sensitivity (93\%). A total of $86 \%$ of patients had abnormal findings between 15 and 20 weeks, which suggest that the diagnosis can be made at the routine anatomic scan. These findings did not differentiate

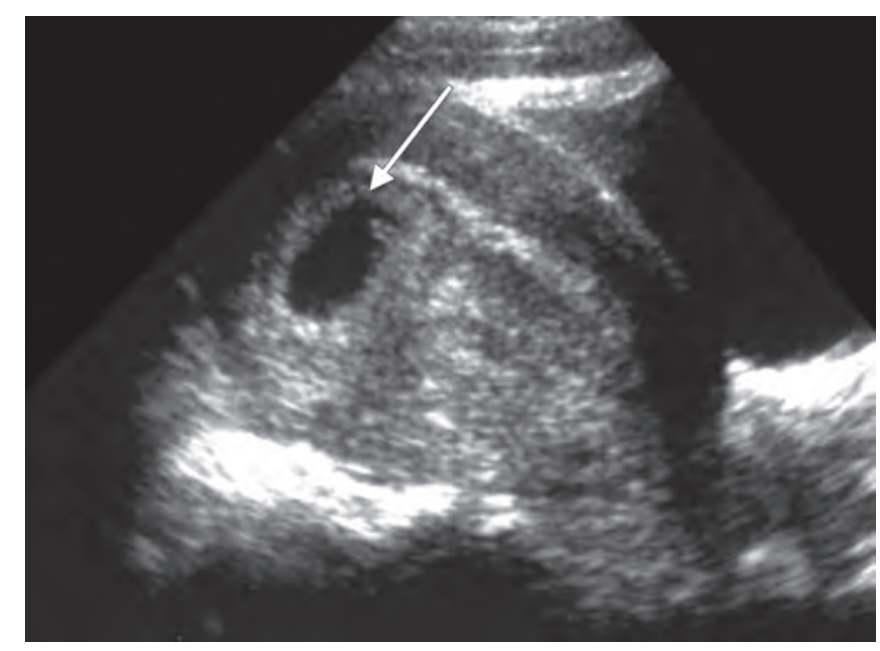

Fig. 2: Normal first-trimester gestational sac

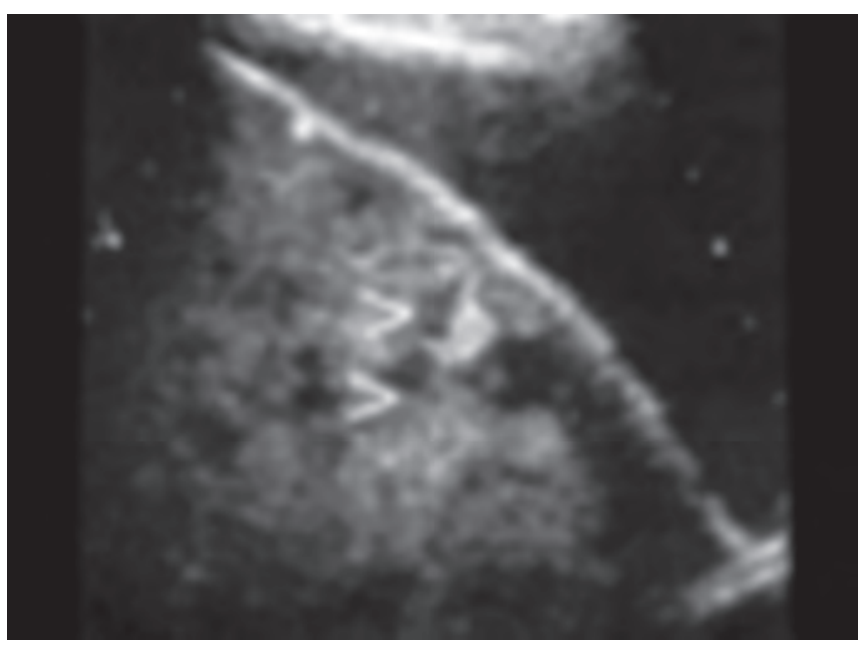

Fig. 4: Interruption of the echogenic line at the uterine bladder interface

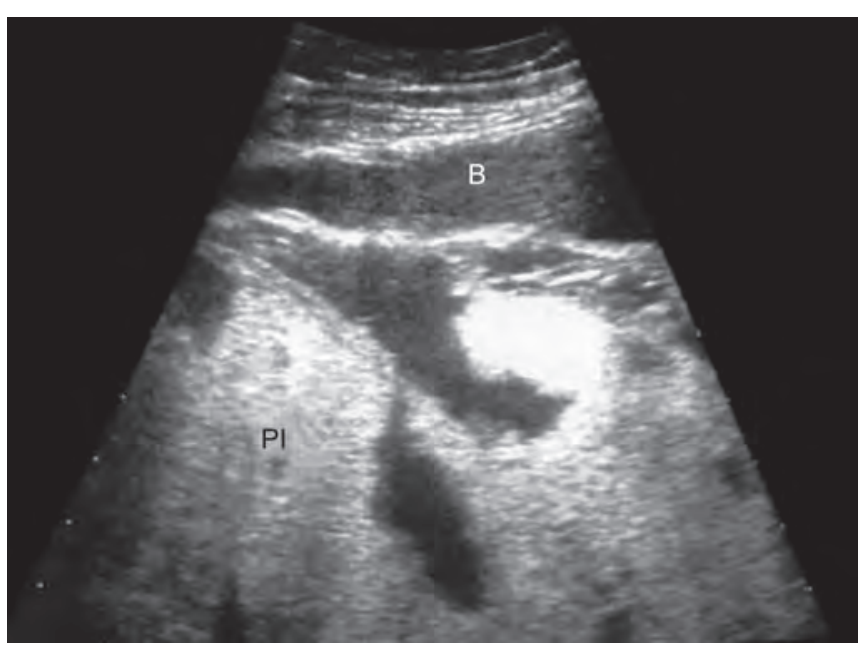

Fig. 6: Obliteration of echolucent area between the uterus and placenta 
Table 3: Sensitivity and positive predictive value (PPV) of sonographic diagnostic criteria for placenta accreta at 15 to 40 weeks

\begin{tabular}{llllll}
\hline & \multicolumn{3}{c}{ Sensitivity } & & \multicolumn{2}{c}{$P P V$} \\
\cline { 2 - 3 } \cline { 6 - 7 } Diagnostic criteria & $n / N$ & $\%$ & & $n / N$ & $\%$ \\
\hline Any criteria & $15 / 15$ & 100 & & $15 / 31$ & 48 \\
$\geq 2$ criteria & $12 / 15$ & 80 & & $12 / 14$ & 86 \\
Lacunae & $14 / 15$ & 93 & & $14 / 15$ & 93 \\
Clear space (isolated) & $1 / 15$ & 7 & & $1 / 17$ & 6 \\
Clear space (with other) & $11 / 15$ & 73 & & $11 / 13$ & 85 \\
Bladder serosa & $3 / 15$ & 20 & & $3 / 4$ & 75 \\
\hline
\end{tabular}

Source: Comstock 2004

between patients with varying degrees of invasion (i.e., accreta $v s$ increta $v s$ percreta).

\section{Three-dimensional (3D) and Color Doppler Sonography}

Three-dimensional ultrasound combined with color flow or power Doppler has been used in the antenatal diagnosis of placenta accretas. Twickler et $\mathrm{al}^{13}$ evaluated 20 out of 215 women who had a prior cesarean delivery and placenta accreta using color flow mapping. Fifteen of these women had cesarean hysterectomies, and nine were found to have placenta accretas. The measurement of $<1 \mathrm{~mm}$ for the smallest myometrial thickness or the presence of large intraplacental lakes was 100\% sensitivity in predicting myometrial invasion (specificity $72 \%$, PPV $72 \%$, negative predictive value (NPV) $100 \%$ ).

Shin et $\mathrm{al}^{14}$ analyzed a total of 170 women, 39 of whom had confirmed placenta accretas at the time of cesarean delivery. Three-dimensional power Doppler was used to assure uteroplacental neovascularization and to depict the abnormal angioarchitecture (Fig. 7). The diagnosis of placenta accreta was regarded as positive if at least one of the following 3D power Doppler criteria was seen on intraplacental hypervascularity; inseparable cotyledonal and intervillous circulation; tortuous vascularity with "chaotic branching" seen on lateral views or numerous coherent vessels involving the serosa-bladder interface seen on a basal view.

Using receiver operative curves, Shih et al showed that the basal view was the best single criterion for the diagnosis of placenta accreta with a sensitivity of $97 \%$ and a specificity of $92 \%$. Using color Doppler criteria, Chou et a ${ }^{15}$ defined five findings that could be considered suggestive of placenta accreta. These were: (1) Dilated vascular channels with diffuse lacunar flow, (2) irregular vascular lakes with focal lacunar flow, (3) hypervascularity linking placenta to bladder, (4) dilated vascular channels with pulsatile venous flow over the cervix, and (5) poor vascularity at sites of loss of the hypoechoic zone. The sensitivity of color Doppler imaging in diagnosing placenta accretas was $82.4 \%$, and the specificity was $96.8 \%$.

Proponents of color Doppler imaging suggest that the technique aids in the abnormal placentation because it can highlight areas of increased vascularity with dilated blood vessels that cross the placenta and uterine wall. Using transabdominal, transvaginal, power Doppler, and magnetic resonance imaging (MRI), Levine et $\mathrm{a}^{16}$ found that power Doppler and MRI offered no increased sensitivity in the diagnosis of placenta accreta in a small series of 19 patients suspected of having abnormal placentation.

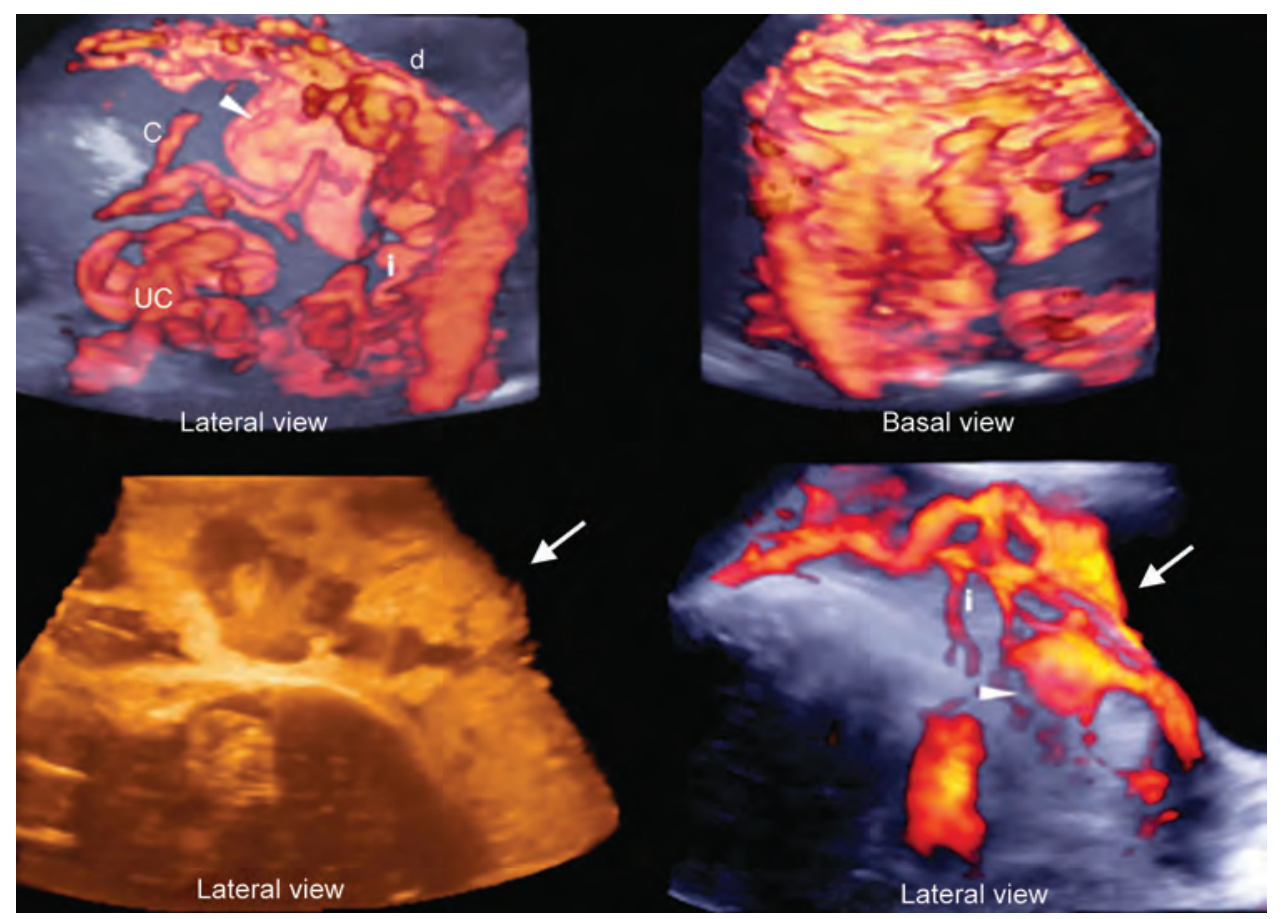

Fig. 7: Three-dimensional color power Doppler of placenta accreta 
They did note that MRI was helpful in diagnosing posterior placenta accretas and concluded that MRI should be reserved for those with a placenta, i.e., difficult to evaluate with ultrasound.

\section{Magnetic Resonance Imaging}

Ultrasound is the primary modality in the evaluation of placenta accreta, but the use of MRI has increased over the last few years as a technique to diagnose and characterize abnormal placentation. Magnetic resonance imaging may be helpful when the placenta is difficult to visualize by ultrasound because of body habitus or if the placenta is implanted posteriorly. The technique also provides greater soft tissue contrast and a larger field of view than can ultrasound. ${ }^{3}$ In addition, MRI can also be useful in cases requiring the assessment of bladder involvement or when ultrasound findings are ambiguous.

One of the first studies to analyze features of placental invasion by MRI was performed by Lax et al. ${ }^{17}$ It was a retrospective review of 20 prenatal MRI studies (10 cases, 10 controls). Three MRI findings were found with abnormal placentation: (1) Abnormal uterine bulging, (2) heterogeneity of signal intensity within the body of the placenta, and (3) the presence of dark intraplacental bands on T2-weighted images. Warshak et al ${ }^{18}$ performed a historical cohort study on 453 women with risk factors for placenta accreta, 39 of whom were found to have pathologically confirmed placenta accreta. They found that ultrasound correctly predicted accreta in 30/39 and ruled out accreta in 398/414 women for a sensitivity of $77 \%$ and a specificity of $96 \%$. Magnetic resonance imaging was used when ultrasound findings were suspicious or inconclusive. Forty-two women underwent MRI, and this technique correctly diagnosed placenta accreta in 23/26 cases and ruled out accretas in 12/14 patients for a sensitivity of $88 \%$ and a specificity of $100 \%$. First, they suggest a two-stage protocol in evaluating a patient at risk of abnormal placentation using ultrasonography and then MRI for cases that are inconclusive. Dwyer et $\mathrm{al}^{19}$ directly compared the sensitivity and specificity of ultrasound vs MRI and found both to be comparable. Ultrasound had sensitivity of $93 \%$ and specificity of $73 \%$ for diagnosing accreta, whereas MRI had a sensitivity of $80 \%$ and a specificity of $65 \%$. In 7 of 32 cases, there was a discordant diagnosis. Sonography was correct in 5 and MRI in 2 of these cases. Their conclusion was that while both ultrasound and MRI are sensitive to diagnosing abnormal placentation, the sensitivities of both methods are not as good as reported in other studies. Their recommendations are to use the other imaging modality if there is an inconclusive finding with the first technique.

The addition of gadolinium-based contrast agents may improve the specificity of MRI, but this is generally considered controversial in pregnancy because of its unknown effects on the fetus. ${ }^{20}$ The material crosses the placenta and is excreted via the fetal kidneys into amniotic fluid. It is a pregnancy class C drug and the American College of Radiology recommends that IV gadolinium should be avoided in pregnancy unless the potential benefit justifies the potential risk to the fetus.

\section{Biological Markers}

Maternal serum alpha-fetoprotein (MSAFP), free beta $h C G$, creatine kinase: Biological markers may have the potential to improve the diagnostic accuracy for placenta accretas. Elevated MSAFP has been reported in retrospective studies performed by both Zelop ${ }^{21}$ and Kupferminc. ${ }^{22}$ In both studies, none of the controls had elevations in MSAFP, whereas half of the accreta cohort did. Hung et $\mathrm{al}^{23}$ also showed that free beta-human chorionic gonadotropin (hCG) levels were elevated (>2.5 M.O.M) in 25\% of patients who were eventually found to have placenta accretas. The breakdown of the fetal maternal placental barrier is the theoretic mechanism for the elevations in both these biological markers. Ophir et $\mathrm{al}^{24}$ published a case report involving two patients with placenta increta and percreta and found both to have elevations in creatine kinase. The postulated mechanism was myometrial muscular damage by the invading trophoblast causing release of creatine kinase into the maternal circulation. There are currently no prospective studies analyzing the role of maternal serum markers and placenta accretas.

Cell-free fetal deoxyribonucleic acid (DNA): Sekizawa et $\mathrm{al}^{25}$ demonstrated higher amounts of cell-free fetal DNA in women with placenta accreta. Maternal blood samples were obtained from 20 patients. Thirteen were controls with male fetuses, and seven patients had placenta previas and risk factors for abnormal placentation. Using real-time qualitative polymerase chain reaction assay to measure fetal DNA in maternal plasma, Sekizawa showed that fetal DNA is increased in cases of placenta previa and more so with placenta accreta (Graph 4). They theorize that the increase is related to the invasion of trophoblasts into the myometrium.

Placenta messenger RNA ( $m R N A$ ): Circulating cell-free placental mRNA can stably be isolated and quantified in maternal plasma. ${ }^{26}$ Miura et al had earlier demonstrated that the levels of placental mRNA could be used to monitor the effect of methotrexate (MTX) treatment for a placenta percreta. In a small study with 24 controls and 4 accretas, they showed that the levels of placental mRNA were significantly higher in maternal plasma. Behery et al used placental mRNA and showed that the levels were significantly higher in patients with placenta 


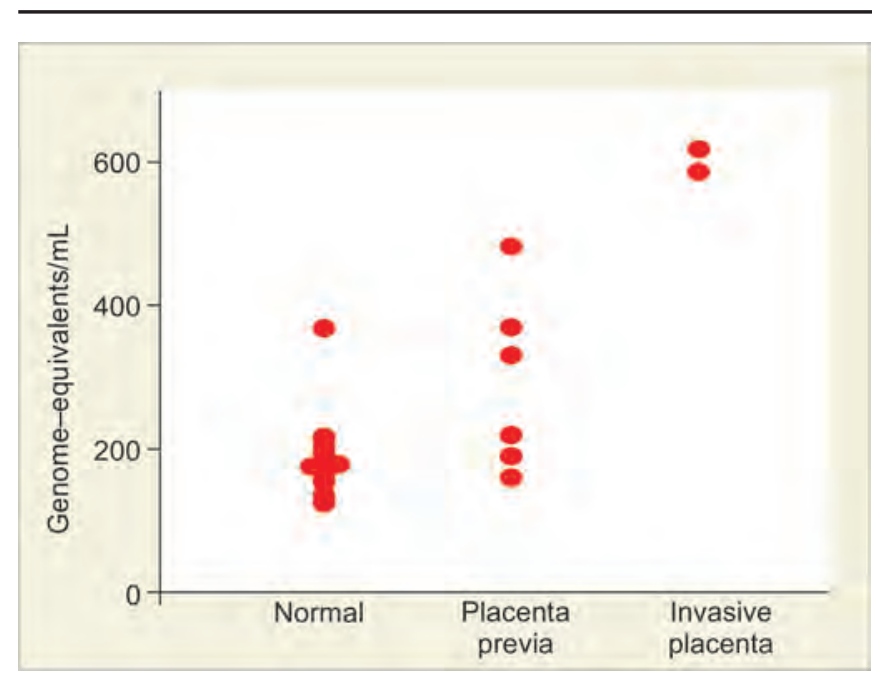

Graph 4: Fetal cell-free DNA concentration in maternal plasma in control and placenta previa/accreta

accreta than in those without placenta accreta. ${ }^{27}$ They concluded that the use of placental mRNA may increase the accuracy of ultrasound and color Doppler in prenatal prediction of placental invasiveness in patients with suspected accretas.

Although promising, the clinical utility of measuring cell-free fetal DNA or placenta mRNA in maternal blood remains uncertain, as does the use of other serum markers, such as MSAFP, free beta hCG, or creatine kinase.

\section{Clinical Outcomes}

Abnormal placentation is associated with significant perinatal morbidity Table 4 . Gielchinsky et $\mathrm{al}^{28}$ performed a case-control analysis of over 34,000 pregnancies from which 310 were diagnosed with placenta accreta.

Most of the accretas in this study were diagnosed during labor so the higher preterm delivery rate was not attributed to a planned earlier delivery. In addition, the neonates had a lower birth weight and were at increased

Table 4: Perinatal outcomes in patients with and without placenta accreta

\begin{tabular}{lllll}
\hline \multicolumn{1}{c}{$\begin{array}{l}\text { Patients } \\
\text { with } \\
\text { accreta } \\
(n=310)\end{array}$} & $\begin{array}{l}\text { Patients } \\
\text { without } \\
\text { accreta } \\
(n=310)\end{array}$ & $\begin{array}{l}\text { Odds ratio (95\% } \\
\text { confidence } \\
\text { interval) }\end{array}$ & $p$-value \\
\hline $\begin{array}{l}\text { Week of } \\
\text { delivery }\end{array}$ & $39.3 \pm 3$ & $40.5 \pm 1$ & & $<0.001$ \\
$\begin{array}{l}\text { Preterm } \\
\text { delivery }\end{array}$ & $33(10.7)$ & $3(1)$ & $12.1(3.7-39.9)$ & $<0.001$ \\
Birth wt (gm) & $3,110 \pm 572$ & $3,408 \pm 415$ & \\
SGA (<10\%) & $83(27.3)$ & $45(14)$ & $5.05(1.46-3.28)$ & $<0.001$ \\
SGA (<5\%) & $48(15.8)$ & $11(3.6)$ & $2.19(2.56-9.90)$ & $<0.001$ \\
Male gender & $139(47.1)$ & $151(50.7)$ & & 0.329 \\
Perinatal & $3(1.0)$ & $0(0)$ & & 0.249 \\
death & & & & \\
\hline
\end{tabular}

Source: Gielchinsky 2004 risk for small for gestational age (SGA). The authors speculate that these findings are related to the pathological implantation of the placenta that interferes with normal placental function resulting in abnormal fetal growth.

Hemorrhage is the most common complication at the time of delivery in a patient with placenta accreta. There is a potential for massive blood loss, resulting in consumptive coagulopathy, renal failure, acute respiratory distress syndrome (ARDS), need for reoperation, and death. ${ }^{3}$ Miller et al showed that the estimated blood loss exceeded 2,000 cc in $66 \%$ of their cohort, $5,000 \mathrm{cc}$ in $15 \%$ of cases, and $10,000 \mathrm{cc}$ in $6.5 \% .{ }^{8} \mathrm{~A}$ total of $55 \%$ of women required transfusion, and $21 \%$ of the patients required more than 5 units of blood.

As the incidence of abnormal placentation has increased, placenta accreta has become one of the leading indications for peripartum hysterectomy. ${ }^{29}$ Glaze et al performed a retrospective review of over 108,000 deliveries in Calgary and noted a peripartum hysterectomy rate of 0.8 per 1,000 deliveries. The leading indication for peripartum hysterectomy was atony (32 out of 87; $37 \%$ ) followed by suspected placenta accreta ( 29 out of $87 ; 33 \%)$. A total of $53 \%$ of the patients ended up needing intensive care unit (ICU) admission postoperatively. Other well-described complications of peripartum hysterectomies for placenta accretas include infection, cystotomy, ureteral injury, and the need for reoperation for hemoperitoneum. ${ }^{8,10}$

\section{Management}

\section{Prenatal Diagnosis and Timing of Delivery}

The preoperative management for a patient with suspected placenta accreta is crucial. The predelivery diagnosis is associated with decreased maternal hemorrhagic morbidity. Warshak et al identified 99 women with placenta accreta in their cohort, 62 of whom were diagnosed predelivery, and 37 of whom were diagnosed intrapartum. The prenatally diagnosed group received fewer units of packed red blood cells (PRBCs) (4.7 units compared with 6.9) and had a lower estimated blood loss (2,344 cc vs $2,951 \mathrm{cc}$ ). The prenatally diagnosed group was delivered electively at 34 and 35 weeks to reduce the morbidity associated with emergent hysterectomy. This practice was associated and was not associated with increased neonatal morbidity (neonatal ICU length of stay, ARDS, need for surfactant administration, or intubation), which may have been related to the increased use of antenatal steroid administration given to the prenatally diagnosed group. Eller et al ${ }^{30}$ also advocate an electively scheduled delivery at 34 weeks after antenatal steroids to decrease morbidity associated with vaginal bleeding and emergent delivery. Their cohort of 69 patients (57 prenatally diagnosed vs 
17 unsuspected) showed that prenatal diagnosis resulted in lower ICU admission rates (23 vs 43\%), lower large volume of blood transfusions (5 vs 9\%), less ureteric injury (5 vs 9\%), less intraabdominal infection (6 vs 9\%), decreased hospital readmission (5 vs 18\%), and less vesicovaginal fistula formation ( 0 vs 6\%). In a meta-analysis of emergent postpartum hysterectomy for hemorrhage, Rossi et $\mathrm{al}^{31}$ noted a mortality rate of $2.6 \%$. Abnormal placentation was the foremost indication for emergent postpartum hemorrhage in this meta-analysis involving about a thousand patients.

\section{Placental Removal}

Eller et $\mathrm{al}^{30}$ also showed that attempts at placental removal prior to hysterectomy had significantly increased early morbidity compared with those who underwent cesarean hysterectomy with the placenta left undisturbed in situ. Kayem et $\mathrm{al}^{32}$ came to the same conclusion in their retrospective review of 31 cases with placenta accreta. Their study compared conservative vs extirpative management of patients with placenta accreta. In the subset of patients who underwent hysterectomies, forced removal of the placenta was associated with massive hemorrhage. Scarantino et $\mathrm{al}^{33}$ described the use of the argon beam coagulator to help achieve hemostasis after the incomplete removal of a posteriorly implanted placenta previa. This case report does note that the attempted removal of the placenta resulted in hemorrhage that resolved with the use of the argon beam. The use of B-lynch sutures, intrauterine balloon, and Affronti square endouterine hemostatic sutures after manual removal has been advocated by Arduini et $\mathrm{al}^{34}$ in a case series of 9 women who underwent postpartum hemorrhage for placenta previa and accreta. The mean decrease in hemoglobin levels in this cohort was $2.4 \mathrm{gm} / \mathrm{dL}$. Their protocol also included preventative catheterization of the descending aorta via transhumeral access to allow embolization of the pelvic vessels though none of their patients required radiological intervention.

\section{Conservative Management}

Cesarean hysterectomy has been the management of choice for placenta accreta. It is associated with significant morbidity as documented above as well as the psychological consequences of the loss of fertility. There are now multiple studies and case reports for the conservative management of abnormal placentation. These include expectant management by leaving the placenta in situ, uterine artery embolization followed by expectant management, and adjuvant medical therapy including MTX, misoprostol, mifepristone, and gonadotropinreleasing hormone analogues.

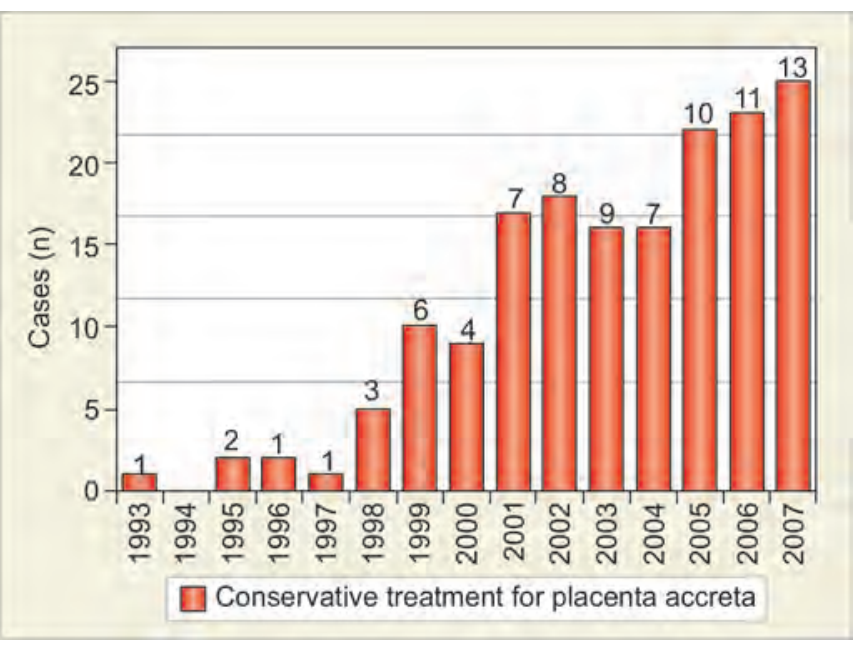

Graph 5: Annual number of accreta cases treated conservatively (Sentilhes 2010)

Sentilhes et $\mathrm{al}^{35}$ recently published a retrospective multicenter cohort study in France assessing maternal outcome after conservative treatment for placenta accreta Graph 5.

Of the 311 women initially studied in this trial, 144 (46.3\%) were excluded because they underwent partial removal of the placenta or cesarean hysterectomy. Conservative treatment was successful in 131 out of 167 women (78.4\%). Thirty-six patients needed a hysterectomy either primarily or delayed. Severe maternal morbidity was noted in 10 patients (6\%). Complications for the conservatively managed cohort included pulmonary edema, septic shock, acute renal failure, infection, deep vein thrombosis or pulmonary embolism, and secondary postpartum hemorrhage. There was one maternal death related to myelosuppression and nephrotoxicity related to MTX administration.

Bretelle et $\mathrm{al}^{36}$ also analyzed 50 cases of placenta accreta, 24 of whom received total hysterectomy, and 26 of whom were managed conservatively. Twenty-four of the conservatively managed group (92\%) received additional treatment (medical, surgical, or embolization), and $19 \%$ needed a hysterectomy. Of this subgroup, there was a $15 \%$ rate of infection and a $12 \%$ rate of disseminated intravascular coagulation (DIC).

Timmermans et $\mathrm{al}^{37}$ retrospectively analyzed 48 case reports or case series of 60 women who underwent conservative management of placenta accretas. Twenty-six women underwent delivery without any additional intervention. Twenty-two of the 26 cases were not diagnosed prenatally, and in three quarters of the cases, the placenta was only partially removed. Four of the 26 failed conservative management and hysterectomies were performed for excessive bleeding or overwhelming infection. Other complications included fever $(2 / 26)$, endomyometritis $(4 / 26)$, and delayed vaginal bleeding $(6 / 26)$. A total 
of 4/26 noted delayed but spontaneous expulsion of placental tissue, $3 / 26$ patients went on to have a subsequent pregnancy, and $2 / 3$ had recurrent abnormal placentation resulting in cesarean hysterectomies.

There are also multiple case reports of the administration of MTX with conservative management of placenta accreta, with the first report published in 1986. Timmermans found that 22 of the 60 women received MTX, some with uterine artery embolization. The failure rate of MTX was 5/22, with failure noted as persistence of vascularized placental tissue, vaginal bleeding, infection, and fever. A total of $8 / 22$ had spontaneous expulsion of the retained placental tissue, and 2/22 had uneventful subsequent pregnancies.

The use of arterial embolization with conservative management was also reviewed by Timmermans, usually immediately after delivery in 12 patients. The failure rate was 3/12, resulting in hysterectomies for infection, vaginal hemorrhage, or DIC. A total of 3/12 patients had spontaneous expulsion of retained placenta, and 3/12 had subsequent pregnancies that were uneventful.

Sentilhes et $\mathrm{al}^{38}$ performed a retrospective cohort study on 100 consecutive women who had postpartum hemorrhage to determine the predictors of failure of embolization. The failure rate of embolization was $11 \%$ for postpartum hemorrhage. Seventeen patients had placenta accretas, and the failure rate of embolization for this subset of patients was 4/17 (24\%). Complications associated with both successful and failed pelvic artery embolization included buttock necrosis requiring surgical debridement, puncture site hematoma, endometritis, postpartum fever, wound infection, postpartum myocarditis and pulmonary embolism.

Provansal et $\mathrm{al}^{39}$ studied fertility and obstetric outcome after conservative management of placenta accreta. Forty-six patients out of a cohort of 73 patients with placenta accreta were treated conservatively without extirpative measures, all of whom had residual placenta in situ. Additional treatments to conserve fertility included bilateral hypogastric artery ligation, uterine artery ligation, uterine sutures, embolization, MTX, oxytocin, and/ or prostaglandins. The median time of follow-up for the cohort was 65 months. The median time for the resumption of menses was 130 days, and none had amenorrhea. A total of 12/14 patients desiring another pregnancy got pregnant for a total of 15 pregnancies, and 2 had recurrent placenta accreta. Five spontaneous abortions were noted, and the median term of delivery was 37 weeks.

\section{Surgical Management}

Eller et al studied 76 cases of placenta accretas. ${ }^{30}$ When accretas were diagnosed prenatally, scheduled cesarean hysterectomies were performed without attempted placental removal. A significant decrease in morbidity was noted in comparison with patients where placental removal was attempted ( $33 \%$ vs $67 \%$; $=0.038)$. The pre- or perioperative use of ureteral stents was noted to decrease the risk of ureteral injury and to significantly reduce the rate of postoperative morbidity $(p=0.02)$. Hypogastric artery ligation was not noted to decrease the mean blood loss or the need for large volume of blood transfusions. They concluded that scheduled cesarean hysterectomy with preoperative ureteral stent placement and avoiding piecemeal placental removal was the optimal management strategy for prenatally diagnosed placenta accretas.

Angstmann et $\mathrm{al}^{40}$ retrospectively analyzed a cohort of 26 cases of placenta accreta; eight cases were managed using a staged embolization procedure with hysterectomy, and outcomes were compared with routine cesarean hysterectomies.

Compared with cesarean hysterectomies, a staged procedure involves preoperative use of interventional radiological embolization followed by hysterectomy, resulting in significant blood loss reductions (553 vs 4517 $\mathrm{mL} ; \mathrm{p}=0.0001)$, need for transfusion (2 vs 16; $\mathrm{p}=0.001$ ), and units of blood transfused ( 0.5 vs $7.9 ; \mathrm{p}=0.0013)$.

Preoperative planning and collaboration can optimize maternal outcomes. In some institutions, delivery in the main operative suites may be more appropriate than a delivery on the labor and delivery unit. Consultation with anesthesia is essential preoperatively. Weiniger et $\mathrm{al}^{41}$ performed a prospective observational study on the anesthetic management of placenta accreta using a high- and low-suspicion classification system. High-suspicion cases included patients with suspicious ultrasound findings as described earlier or bleeding placenta previa with a prior uterine scar. Low suspicious cases included anterior placentas without prior cesarean surgery or nonbleeding placenta previa without ultrasound findings but one previous cesarean section. A total of 12/17 (71\%) of accretas were correctly diagnosed using the high-risk system. Unfortunately, 2/11 (18\%) of the low-risk group had undiagnosed accretas. All high-risk patients underwent general anesthesia and all patients with accretas ended up with cesarean hysterectomies. Despite missing two accretas with the low-risk classification system, neither patient received many blood transfusions nor underwent emergent hysterectomies. There were significant differences in both groups with the use of general anesthesia, invasive systemic blood pressure monitors, large-bore intravenous cannulas prior to the start of surgery, fresh frozen plasma, platelets, cryoprecipitate, and the use of crystalloids. There were no differences in the rate of blood transfusions and postoperative renal function and 
coagulopathy. In a review of the anesthetic management of abnormal placentation, Kuczkowski ${ }^{42}$ notes that there is a high rate of conversion of neuraxial anesthesia to general anesthesia (28\%) because of inadequate operating conditions and/or patient discomfort. He also notes that there is a high rate of DIC (27\%). The anesthetic recommendations include the use of two large-bore intravenous catheters, arterial lines, and possibly central lines preferable prior to the start of the cesarean section. Vasoactive drugs (phenylephrine, ephedrine, dopamine, or epinephrine) and PRBCs should be immediately available. Consideration should be given to the use of cell saver and acute normovolemic hemodilution.

The bladder is the most common affected pelvic structure with placenta percreta with ureteral and ileal involvement also reported. ${ }^{42}$ The occurrence of bladder invasion is estimated to be $1 / 10,000$ births. Gross hematuria occurs in about $25 \%$ of such cases. $\mathrm{Ng}$ et al reviewed 5 cases, 2 of whom had undergone urologic assessment preoperatively (including pelvic ultrasound and MRI).

Preoperative urologic management included cystoscopy and ureteral stent placement resulting in no urologic complications postoperatively, despite urgent timing in both cases. The three cases who were assessed intra- or postoperatively all encountered urologic complications including bladder injury, ureteral injury, and massive blood transfusions (12-44 units). Konijeti et al ${ }^{43}$ recom- mend making every attempt to preserve the bladder after proving the integrity of the ureters. Reconstructive surgery can be postponed until after the patient is hemodynamically stabilized. Anterior bladder dissection is helpful for defining dissection planes and determining whether posterior bladder wall resection is required. The resection of the bladder base carries with it the risks of coagulopathy, ARDS, multiorgan failure, and vesicovaginal fistula due to blood loss and extensive surgery.

\section{ETIOLOGY}

There is good evidence that placenta accreta forms due to the failure of a normal deciduas to form, either because the endometrium is deficient or because it cannot transform. ${ }^{44}$ Placenta accreta is a regular finding in abdominal and ectopic pregnancies where there is also no normal endometrium to transform into decidua.

The placenta forms differently from other organs. It is created by cellular interactions of maternal cells and fetal trophoblastic cells, each directed by different genomes. ${ }^{45}$ The placental interaction with endometrium begins at implantation. Early on, trophoblasts invade the veins and stroma of maternal tissues, allowing the placenta to grow into the uterine cavity. Nitabuch's fibrinoid lies between the placenta and the uterine tissues. It is an amorphous eosinophilic matrix containing trophoblastic cell proteins and maternal fibrin (Fig. 8).

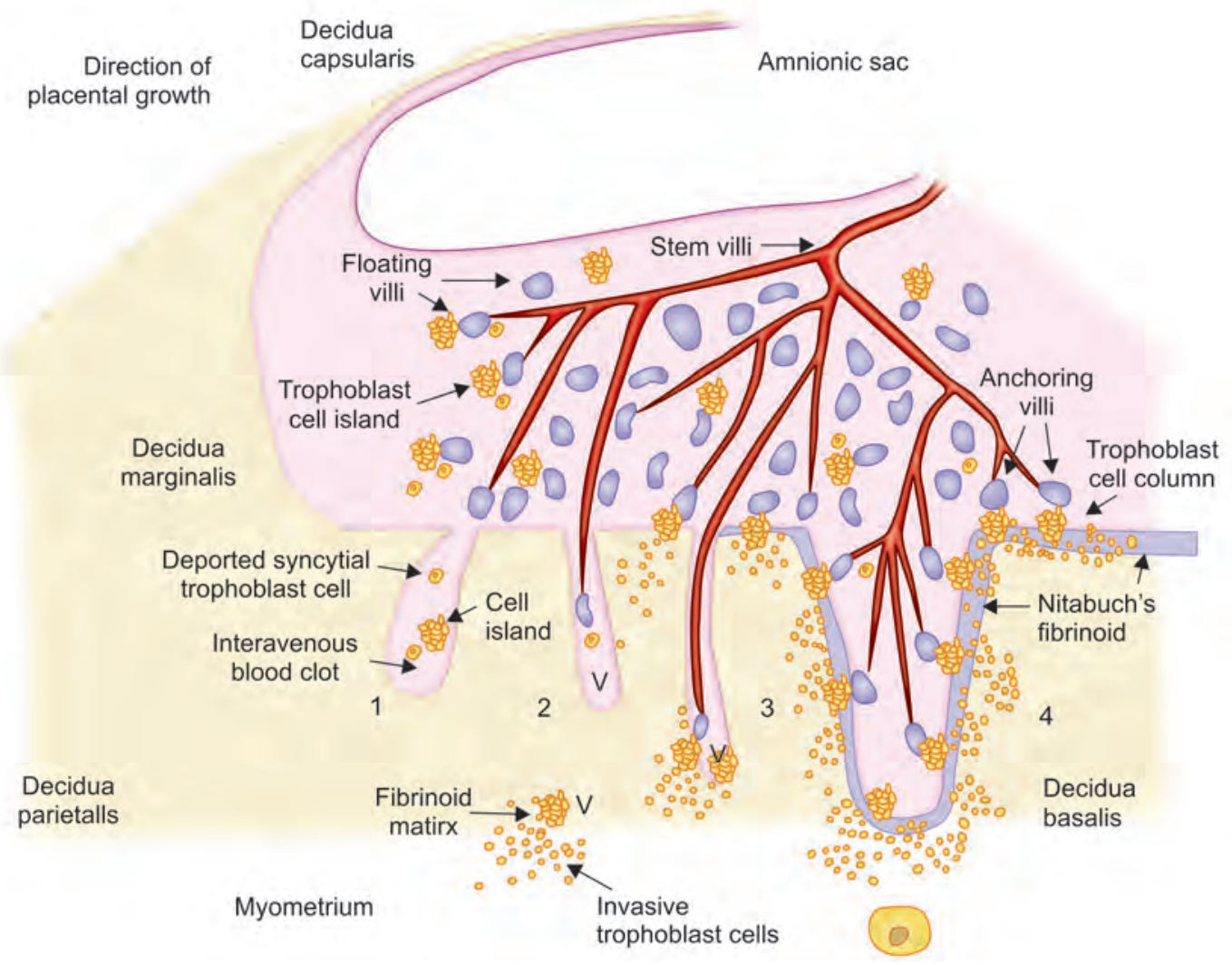

Fig. 8: Normal placental implantation 
Without a normal decidual plate and Nitabuch's layer, the villous trophoblast has direct access to the maternal myometrium. However, accreta is often focal, such that next to areas of abnormal adherence with no decidua, there can be an area of normal decidua basalis, and it is this layer that allows normal separation to take place after birth. ${ }^{44}$ Stanek et $\mathrm{al}^{46}$ coined the term "occult placenta accreta" (OPA), which others refer to as mild or focal placenta accreta. They found a statistical association of OPA with indicators of uteroplacental hypoxia including decidual hemosiderosis. All this points to evidence of a trophoblast/decidual antagonism existing on a molecular level, with the decidua having an impact on trophoblast differentiation. Earl et $\mathrm{al}^{47}$ have shown that placenta accreta is not related to excessive trophoblastic invasiveness with respect to trophoblastic production of human placental lactogen, hCG, and pregnancy-specific $\beta 1$-glycoprotein and that the absence of deciduas is more important in the pathogenesis of placenta accretas. Tseng et $\mathrm{al}^{48}$ theorize that trophoblastic migration and invasion during normal placental development must be interdependently influenced by different kinds of molecules, such as growth factors and their receptors, cytokines, hormones, adhesion molecules, and enzymes in an autocrine or paracrine manner, and that normal placentas do not proceed beyond the inner third of the myometrium through tight spatial and temporal regulation.

Tyrosine kinase receptors are believed to be involved in placental cellular proliferation, migration, and differentiation. Epidermal growth factor receptor (EGFR) and the c-erbB-2 oncoprotein are tyrosine kinase receptors that are functionally related and expressed in high amounts in human placentas. Tseng et al ${ }^{49}$ have shown that EGFR is expressed in significantly higher amounts in placenta accretes, while the immunoreactivity of c-erbB-2 was significantly lower, concluding that the development of accretas is related closely to the differential expression of these proteins.

Placental accreta is characterized by profuse uteroplacental neovascularization. Vasculoendothelial growth factor (VEGF), placenta growth factor (PlGF), and their receptors are well-known angiogenic factors and during normal pregnancy, the expression of VEGF and its receptors in trophoblasts regulates the secretion of many proteins and hormones via both autocrine and paracrine regulation. ${ }^{48}$ Tseng et $\mathrm{al}^{50}$ showed via enzyme-linked immunosorbent assay (ELISA) of placental lysates that placenta accretas had significantly higher VEGF and lower soluble vasculoendothelial growth receptors-2 (VEGR-2) compared with normal controls. In addition, they showed that PlGF and sVEGR-1 levels were not different in accretas and controls. The increased VEGF and decreased sVEGR-2 imply paracrine induction of neovascularization during the development of accretas.

Angiopoietin and Tie receptors have roles in vasculogenesis and angiogenesis and have been well studied in malignancies. Tie- 1 is a tyrosine kinase with immunoglobulin and epidermal growth homology domains, while Tie-2 is a tunica internal endothelial cell kinase receptor. Both are graded as specific vascular endothelial markers, and both are also expressed in the placenta. ${ }^{51}$ Using Western blot and ELISA analysis, placenta accretas were shown to have higher angiopoietin-2 and lower Tie-2 than in normal controls and that life-threatening uteroplacental neovascularization may be correlated with these molecular changes. The decreased Tie- 2 expression might imply a negative feedback response rather than a primary event during the formation of an accreta's vascular network.

Extracellular matrix proteolysis plays a major role in trophoblast invasion. The zinc requiring matrix metalloproteinase (MMP) family is responsible for matrix degradation and plays a role in angiogenesis. ${ }^{48}$ The four main groups are collagenases, gelatinases, stromelysins, and membrane-type MMPs. Collagenases degrade fibrillar collagens I, II, and III and include collagenase (MMP-1), neutrophil collagenase (MMP-8), and collagenase 3 (MMP-13). ${ }^{52}$ Gelatinases degrade denatured collagens, collagen IV, and include gelatinase A (MMP-2) and gelatinase B (MMP-9). Amnion, basal plate, chorionic plate, deciduas, chorion laeve, Nitabuch's fibrinoid, umbilical cord, and placenta villi almost show immunoreactivity for MMPs. Demir-Weusten et a ${ }^{53}$ performed immunohistochemistry and zymography on placental tissue sections and protein extracts and showed that MMP-2 activity is highest in areas where trophoblast cells invade maternal tissues. Matrix metalloproteinases-9 had the highest enzymatic activity where fetal and maternal parts contacted, suggesting a role in the separation of the placenta from the uterine wall during normal labor. Matrix metalloproteinases-3 had similar localization to MMP-9, which also suggested a role for this enzyme during labor. Matrix metalloproteinases are regulated at (1) a transcriptional level depending on gene expression via factors like hormones, cytokines, growth factors; (2) a secretion level depending on packaging in secretion granules; and (3) at the protein level depending on activation or inhibition of a proform and via stabilization via glycosylation. Cohen et al ${ }^{52}$ suggest that MMP-9 may play a more important role than MMP-2 in trophoblastic invasion during pregnancy though Staun-Ram et al ${ }^{54}$ suggest that MMP-2 represents the main gelatinase early at implantation in pregnancy but that by the end of the first trimester both MMP-2 and MMP-9 are involved. MMP-26 proteolyzes fibrinogen and extracellular matrix proteins and probably plays a role in tissue remodeling 
during placentation. MMP-14 and MMP-15 are also present in the first trimester and act as activators of MMP-2. Other MMPs have been found in trophoblasts including MMP-1, MMP-3, MMP-7, MMP-18, and MMP-19, but their role in placental invasion is not clear.

The role of decidua in preventing abnormal placentation is probably by an autocrine/paracrine feedback. Hu et a ${ }^{55}$ have confirmed that decidual natural killer cells (dNK) play an important role in the immune regulation of trophoblast invasion. Using decidual tissue from elective terminations of pregnancies, $\mathrm{Hu}$ showed that dNK inhibited trophoblastic migration via soluble mediators, specifically interferon gamma. In addition, the inhibition of trophoblastic migration was dose dependent, indicating that trophoblastic control was mediated by soluble factors produced by dNK.

Insulin and the insulin-like growth factors belong to a family of peptides essential for fetal growth and development. Relaxin belongs to the insulin-like growth factor family and may also play a role as a regulator of cell growth in fetal membranes and placenta via autocrine/paracrine interactions and its site of action in the uterus has been shown to be primarily in the decidua. ${ }^{56}$ Another insulin like growth factor INSL4 (Insulin-like 4) has been shown to play a role in the concerted regulation of placental development and fetal growth via apoptosis. Goh et al ${ }^{58}$ showed by gene expression and immunohistochemistry that INSL4 expression was significantly decreased in trophoblastic tissue in both invasive and non-invasive areas in placenta accreta when compared to gestational aged matched controls suggesting accreta formation is not a local trophoblastic problem but a more generalized phenomenon suggesting that the pathophysiology of TR invasiveness is related to reduced TR apoptosis. ${ }^{30}$ This group also showed that increased Relaxin gene and protein expression was associated with significant antepartum bleeding in accreta patients.

\section{CONCLUSION}

The incidence of placenta accreta is rising with the rates as high as 1/533 pregnancies. Accreta are now the chief cause of postpartum hemorrhage and are a significant cause of both maternal and neonatal morbidity and occasionally mortality. The main risk factor for the development of placenta accreta is a history of a prior cesarean delivery. The prenatal diagnosis of accreta is crucial and is associated with a significant reduction in maternal blood loss of post partum complications. Ultrasound findings suggestive of accreta include placental lacunae, myometrial thinning to less than $1 \mathrm{~mm}$, the loss of a placental-uterine interface and an abnormal uterine bladder interface. The use of color and 3D Doppler ultrasound is also helpful in diagnosing placenta accreta. The trend toward conservative and management of placenta accreta using embolization techniques, methotrexate and observation must be balanced with a significant rate of complications, such as infection and DIC. Histologically, the absence of a maternal decidual layer allows placental trophoblastic tissue to invade into the maternal myometrium. Many peptides and growth factors have been examined in placenta accreta and there is likely a defect in the paracrine/autocrine feedback between the placental trophoblast and maternal decidua. The interaction of hormones, such as Relaxin which is abundant in maternal decidua and INSL4, an insulin like peptide found in placental trophoblastic tissue may play role in the formation of placenta accreta.

\section{REFERENCES}

1. MMWR Morb Mortal Wkly 1998; 47:705-707.

2. Berg C, Chang J, Callaghan W, et al. Pregnancy related mortality in the United States, 1991-1997. Obstet Gynecol 2003;101: 289-297.

3. Bauer S, Bonnano C. Abnormal Placentation. Semin Perinatol 2009;33:88-96.

4. Hull A, Resnik R. Placenta Accreta and Postpartum Hemorrhage. Clin Ob/Gyn 2010;53(1):228-236.

5. ACOG Committee opinion, Number 266 Jan 2002; placenta accreta. Obstet Gynecol 2002;99:169-170.

6. Warshak C, Ramos G, Eskdander R, et al. Effect of predelivery diagnosis in 99 consecutive cases of placenta accreta. Obstet Gynecol 2010;115(1):65-69.

7. Wu S, Kochergnisky M, Hibbard J. Abnormal placentation: twenty year analysis. Am J Obstet Gynecol 2005;192:1458-1461.

8. Miller D, Chollet J, Murphy T. Clinical risk factors for placenta previa-placenta accreta. Am J Obstet Gynecol. 1997; 177:210-214.

9. Zaideh S, Abu-Heija A, El-Jallad M. Placenta praevia and accreta: analysis of a two year experience. Gynecol Obstet Invest 1998;46:96-98.

10. Silver R, Landon M, Rouse D, et al. Maternal Morbidity Associated with Multiple Repeat Cesarean Deliveries. Obstet Gynecol 2006;107:1226-1232.

11. Comstock C, Lee W, Vettraino I, et al. The early sonographic appearance of placenta accreta. J Ultrasound Med 2003 Jan;22(1):19-23.

12. Comstock C, Love J, Bronsteen R, et al. Sonographic detection of placenta accreta in the second and third trimester. Am J Obstet Gynecol 2004;190(4):1135-1140.

13. Twickler D, Lucas M, Balis A, et al. Color flow mapping for myometrial invasion in women with a prior cesarean delivery. J Matern Fetal Med 2000;9(6):330-335.

14. Shih J, Palacios J, Su Y, et al. Role of three-dimensional power Doppler in the antenatal diagnosis of placenta accreta: comparison with gray-scale and color Doppler techniques. Ultrasound Obstet Gynecol 2009;33:193-203.

15. Chou M, Ho E, Lee Y. Prenatal diagnosis of placenta previa accreta by transabdominal color Doppler ultrasound. Ultrasound Obstet Gynecol 2000;15:28-35.

16. Levine D, Hulka C, Ludmir J, et al. Placenta accreta: Evaluation with Color Doppler US, Power Doppler US, and MR Imaging. Radiology 1997;205:773-776.

17. Lax A, Prince M, Mennitt K, et al. The value of specific MRI features in the evaluation of suspected placenta accreta. Magn Reson Imagin 2007;25(1):87-93. 
18. Warshak C, Eskander R, Hull A, et al. Accuracy of ultrasonography and magnetic resonance imaging in the diagnosis of placenta accreta. Obstet Gynecol 2006;108(3):573-581.

19. Dwyer B, Belogolovkin V, Tran L, et al. Prenatal diagnosis of placenta accreta: sonography or magnetic resonance imaging. J Ultrasound Med 2008;27(9):1275-1281.

20. Ophir E, Singer-Jordan J, Odeh M, et al. Abnormal placental invasion-a novel approach to tdreatment. Ob Gyn Survey 2009;64(12):811-822.

21. Zelop C, Nadel A, Frigoletto F, et al. Placenta accreat/ percreta/increta: a cause of elevated maternal serum alphafetoprotein. Obstet Gynecol 1992;80(4):63-64.

22. Kupferminc M, Tamura R, Wigton T, et al. Placenta accreta is associated with elevated maternal serum alpha-fetoprotein. Obstet Gynecol 1993;82(2):266-269.

23. Hung T, Shau W, Hsieh C, Chiu T, Hsu J, Hsieh T. Risk factors for placenta accreta. Obstet Gynecol. 1999 Apr:93(4):545-550.

24. Ophir E, Tendler R, Odeh M, Khouri s, Oettinger M. Creatine kinase as a biochemical marker in diagnosis of placenta increta and percreta. Am J Obstet Gynecol. 1999 Apr;180(4):1039-1040.

25. Sekizawa A, Jimbo M, Iwasaki, et al. Increased cell-free fetal DNA in plasma of two women with Invasive Placenta. Clin Chem 2002;4(2):353-354.

26. Miura K, Miura S, Yamasaki K, et al. Increased level of cell-free placental mRNA in a subgroup of placentda previa. Prenat Diagn 2008;28(9):805-809.

27. Behery M, Rasha E, El Alfy Y. Cell-free placental mRNA in maternal plasma to predict placental invasion in patients with placenta accreta. Int J Gynecol Obstet 2010;109:30-33.

28. Gielchinsky Y, Mankuta D, Rojansky N, et al. Perinatal outcome of pregnancies complicated by placenta accreta. Obstet Gynecol 2004;104(3):527-530.

29. Glaze S, Ekwalanga P, Roberts G, et al. Peripartum hysterectomy: 1999 to 2006. Obstet Gynecol 2008; 111(3):732-738.

30. Eller A, Porter T, Soisson P, Silver R. Optimal management strategies for placenta accreta. BJOG 2009;116(5):648-654.

31. Rossi A, Lee R, Chmait R. Emergency postpartum hemorrhage for uncontrolled postpartum bleeding. Obstet Gynecol 2010;115(3):637-644.

32. Kayem G, Davy C, Goffinet F, et al. Conservative versus extirpative management in cases of placenta accreta. Obstet Gynecol 2004;104(3):531-536.

33. Scarantino S. Reilly J, Moretti M, Pillari V. Argon beam coagulation in the management of placenta accreta. Obstet Gynecol 1999;94(2):825-827.

34. Arduini M, Epicoco G, Clerici G, et al. B-Lynch suture, intrauterine ballon, and endouterine hemostatic suture for the management of postpartum hemorrhage due to placenta previa accreta. Int J Gynecol Obstet 2010;108(3):191-193.

35. Sentilhes L, Ambroselli C, Kayem G, et al. Maternal outcome after conservative treatment of placenta accreta. Obstet Gynecol 2010;115(3):526-534.

36. Bretelle F, Courbiere B, Mazouni C, et al. Management of placenta accreta: morbidity and outcome. Eur J Obstet Gynecol Reprod Biol 2007;133(1):34-39.

37. Timmermans S, van Hof A, Duvekot J. Conservative management of abnormally invasive placentation. Obstet Gynecol Surv 2007;62(8):529-539.

38. Sentilhes L, Gromez A, Clavier E, et al. Predictors of failed pelvic arterial embolization for severe postpartum hemorrhage. Obstet Gynecol 2009;113(5):992-999.

39. Provansal M, Courbiere B, Agostini A, et al. Fertility and obstetric outcome after conservative management of placenta accreta. Int J Gynecol Obstet 2010:109(2):147-150.
40. Angstmann T, Gard G, Harrington T, et al. Surgical management of placenta accreta: a cohort series and suggested approach. Am J Obstet Gynecol 2010;202(1):28:e1-9.

41. Weiniger C, Elram T, Ginorsar Y, et al. Anaesthetic management of placenta accreta: use of a pre-operative high and low suspicioun classification. Anaesthesia 2005;60:1079-1084.

42. Kuczkowski K. Anesthesia for the repeat cesarean section in the parturient with abnormal placentation: what does an obstetrician need to know? Arch Gynecol Obstet 2006;273(6): 319-321.

43. Konijeti R, Rajfer J, Askari A. Placenta percreta and the Urologist. Rev Urol 2009;11(3):173-176.

44. Bernirschke K, Kaufmann P. Pathology of the human placenta. New York; Springer-Verlag 2006.

45. Craven C, Chedwick L, Ward K. Placental basal plate formation is associated with fibrin deposition in decidual veins at sites of trophoblastic cell invasion. Am J Obstet Gyneceol 2002;186(2):291-296.

46. Stanek J, Drummond Z. Occult Placenta Accreta: the missing link in the diagnosis of abnormal placentation. Ped Dev Path 2007;10:266-277.

47. Earl U, Bulmer J, Briones A. Placenta accreta: an immunohistologic study of trophoblast populations. Placenta. 1987 May-Jum; 8(3):273-282.

48. Tseng J, Chou M. Differential Expression of Growth-, Angiogenesis- and Invasion-related Factors in the Development of Placenta Accreta. Taiwanese J Obstet Gynecol 2006;45(2): 100-105.

49. Tseng J, Hsu S, Wen M, et al. Expression of epideral growth factor receptor and c-erbB-2 oncoprotein in trophoblast populations of placenta accreta. Am J Obstet Gynecol 2004;191: 2106-2113.

50. Tseng J, Chou M, Hsieh Y, et al. Differential expression of vascular endothelial growth factor, placenta growth factor and their receptors in placentae from pregnancies complicated by placaenta accreta. Placenta 2006;27:70-78.

51. Tseng J, Hsu S, Ho E, Hsieh Y, Wen M, Chou M. Differential expression of angiopoietin-1, angiopoietin-2 and Tie receptors in placentas from pregnancies complicated by placenta accreta. Am J Obstet Gynecol 2006;194:564-571.

52. Cohen M, Meisser A, Bischof P. Metalloproteinases and human placenta invasiveness. Placenta 2006;27:783-793.

53. Demir-Weusten A, Seval Y, Kaufmann P, Demir R, Yucel G, Huppertz B. Marix metalloproteinases-2, -3 and -9 in human term placenta. Acta histochemica 2007;109:403-412.

54. Staun-Ram E, Goldman S, Gabarin D, Shalev E. Expression and importance of matrix metalloproteinase 2 and 9 (MMP-2 and -9) in human trophoblast invasion. Reprod Biol Endocrinol 2004;2:59.

55. Hu Y, Dutz J, MacCalman C, et al. Decidual NK cells alter in vitro first trimester extravillous cytotrophoblast migration: a role for IFN-gamma. J Immunol 2006;15:177(12):8522-8530.

56. Bryant-Greenwood G, Yamamoto S, Lowndes K, et al. Human decidual relaxin and preterm birth. Ann. N Y Acad Sci 2005;1041:338-344.

57. Millar L, Streiner N, Webster L, et al. Early placental insulinlike protein (INSL4 or EPIL) in placental and fetal membrane growth. Biol Reprod 2005;73:695-702.

58. Goh W, Yamamoto S, Thompson K, Bryant-Greenwood G. Relaxin, its receptor (RXFP1), and insulin-like peptide 4 expression through gestation and in placenta accreta. Reprod Sci 2013;20(8):968-980. 